\title{
SLM phase mask optimization for fiber OAM mode excitation
}

\author{
Astorino, Antonio; Bañas, Andrew Rafael; Rottwitt, Karsten; Glückstad, Jesper
}

Published in:

Proceedings of SPIE

Link to article, DOI:

$10.1117 / 12.2507424$

Publication date:

2019

Document Version

Publisher's PDF, also known as Version of record

Link back to DTU Orbit

Citation (APA):

Astorino, A., Bañas, A. R., Rottwitt, K., \& Glückstad, J. (2019). SLM phase mask optimization for fiber OAM mode excitation. In J. Glückstad, D. L. Andrews, \& E. J. Galvez (Eds.), Proceedings of SPIE (Vol. 10935). [109350F] SPIE - International Society for Optical Engineering. Proceedings of SPIE - The International Society for Optical Engineering https://doi.org/10.1117/12.2507424

\section{General rights}

Copyright and moral rights for the publications made accessible in the public portal are retained by the authors and/or other copyright owners and it is a condition of accessing publications that users recognise and abide by the legal requirements associated with these rights.

- Users may download and print one copy of any publication from the public portal for the purpose of private study or research.

- You may not further distribute the material or use it for any profit-making activity or commercial gain

- You may freely distribute the URL identifying the publication in the public portal 


\section{SLM phase mask optimization for fiber OAM mode excitation}

\section{Antonio Astorino, Andrew R. Bañas, Karsten Rottwitt, Jesper Glückstad}

Antonio Astorino, Andrew R. Bañas, Karsten Rottwitt, Jesper Glückstad, "SLM phase mask optimization for fiber OAM mode excitation," Proc. SPIE 10935, Complex Light and Optical Forces XIII, 109350F (1 March 2019); doi: $10.1117 / 12.2507424$

SPIE. Event: SPIE OPTO, 2019, San Francisco, California, United States 


\title{
SLM phase mask optimization for fiber OAM mode excitation
}

\author{
Antonio Astorino, Andrew R. Bañas, Karsten Rottwitt, and Jesper Glückstad \\ DTU Fotonik, Ørsteds Plads, Building 343, 2800 Kongens Lyngby, Denmark
}

\begin{abstract}
Fiber orbital angular momentum (OAM) modes can be employed in mode-division multiplexing to increase the channel capacity in optical communication systems. Over the years, several experiments to excite high-purity OAM modes by using one phase-only spatial light modulator (SLM) have been conducted. Since phase-only SLMs are intrinsically imperfect for this purpose due to the impossibility to simultaneously modulate both amplitude and phase in the light source, optimal phase masks need to be generated by iterative algorithms. However, if the state of every pixel in the mask is an unknown of the problem, the computational cost is extremely high. The system circular symmetry can be exploited to overcome this issue. Here, for the first time, this approach is implemented and a simple machine learning algorithm is developed to calculate optimal phase masks with a low number of unknowns and iterations. Simulated and experimental results show that the developed technique is capable of exciting high-purity OAM modes.
\end{abstract}

Keywords: orbital angular momentum of light, fiber mode excitation, gradient descent, spatial light modulator, machine learning

\section{INTRODUCTION}

The scientific research dedicated to mode-division multiplexing has recently led to the exploitation of the orthogonality of fiber OAM modes. ${ }^{1-4}$ These modes can be excited by illuminating one fiber end with a properly tailored light beam. One method to create such a beam is by means of a phase-only SLM. Assuming a perfect cylindrical symmetry in the system, the phase contribution given by the SLM phase mask can be expressed, in polar coordinates $(r, \phi)$, as $\exp (i \nu \phi+i \psi(r)), i$ being the imaginary unit, $\nu$ the OAM topological charge, and $\psi(r)$ a real function that needs to be found. ${ }^{5}$

Although a practical method has already been proposed to intrinsically calculate $\psi(r)$ and to optimize some free setup parameters, ${ }^{6}$ it is not demonstrated that the resulting phase mask is optimal. Moreover, the parameters calculated using the algorithm proposed in Ref. 6 may differ from those adopted in the lab due to inaccuracies and uncertainties. For example, the source intensity and phase profiles, relevant distances between optical components, or the illumination beam divergence may not be accurately measurable. Therefore, a phase mask calculated through simulated ideal scenarios requires fine adjustments when used in actual experiments. Intuitively, this entails that the state of each pixel in the SLM phase mask is one unknown of the numerical problem. However, since the number of pixels is usually very large (of the order of $10^{6}$ ), the computational cost is extremely high. As pointed out in Ref. 5, the assumption of a perfect axial alignment in the setup and the consequent system rotational symmetry can be exploited to drastically simplify the problem. The phase mask is divided into $N<100$ contiguous annular regions fully defined by the sequence $\left(\psi_{n}\right)_{n \in[1, N]}$, corresponding to a discretized version of $\psi(r)$. As a result, the number of unknowns is reduced to $N$, namely 4 orders of magnitude less than its original value.

In this work, the problem of finding $\left(\psi_{n}\right)_{n \in[1, N]}$ such that the targeted mode is excited with the highest possible purity is addressed. A method designed for this purpose is presented. Its validity is demonstrated through simulated and experimental results.

Further author information: (Send correspondence to A.A.)

A.A.: E-mail: antonio.astorino.ing@gmail.com, Telephone: +45 50221061

Complex Light and Optical Forces XIII, edited by Jesper Glückstad, David L. Andrews,

Enrique J. Galvez, Proc. of SPIE Vol. 10935, 109350F · (c) 2019 SPIE

CCC code: $0277-786 \mathrm{X} / 19 / \$ 18 \cdot$ doi: $10.1117 / 12.2507424$

Proc. of SPIE Vol. 10935 109350F-1 


\section{PROBLEM DESCRIPTION}

\subsection{Basic Configuration for OAM Mode Excitation}

The setup in Fig. 1 is used to excite a given OAM mode. The linearly polarized Gaussian beam produced by a laser source is shone onto the SLM. Omitting the time dependence of the field for simplicity, at the SLM plane, the electric field of the beam is described by

$$
E_{s}=E_{s, 0} \exp \left(\frac{-r^{2}}{w_{s}^{2}}\right)
$$

where $E_{s, 0}$ is a complex constant and $w_{s}$ is the beam radius. The polarization of the source is parallel to the liquid crystal molecules of the SLM. The (ideally) flat light beam wavefront of $E_{s}$ is modulated by the SLM. The resulting beam propagates in free space and impinges on the quarter-wave plate. The axis of the wave plate is at $\pm 45^{\circ}$ with respect to the polarization direction of the beam. The sign of the angle is chosen according to the sign of the OAM topological charge of the target mode, as discussed in Ref. 6. At this point, the beam is circularly polarized $(\mathrm{CP})$. The circular aperture acts as a spatial filter, whose role will be clarified later. The aperture can be placed anywhere in the free-space path. The lens, positioned at a distance $d_{\ell}$ from the SLM, couples the obtained beam into the fiber.

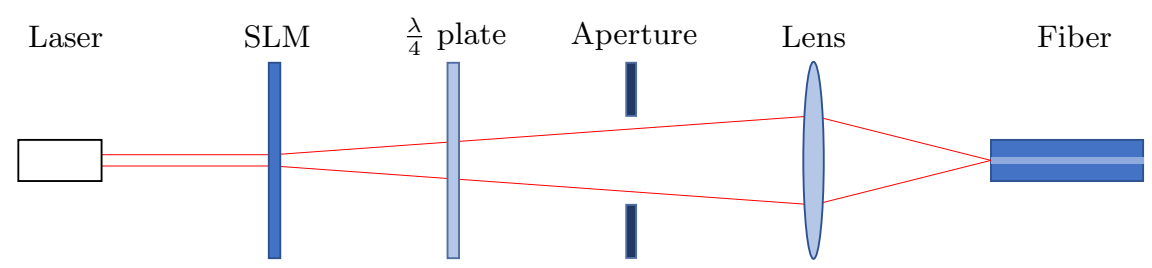

Figure 1: Basic schematic for generating one fiber mode in free space by means of an SLM and lens.

As already mentioned, the use of a quarter-wave plate to generate full-vectorial modes entails that such modes are CP. However, in any cylindrical waveguide, the transverse field of full-vectorial hybrid modes is, in general, locally elliptically polarized. ${ }^{7}$ This means that the field produced using the schematic in Fig. 1 cannot excite pure OAM modes. Nevertheless, using the circularly-polarized-mode approximation described in Ref. 6, the discrepancy between OAM modes and their approximation to CP modes is minimized.

\subsection{Initial Phase Mask and Parameter Calculation - Lossless Optimization}

Let $E_{f}$ be the electric field produced at the fiber end and $U_{f}$ be the polarization component of the calculated CP mode parallel to the beam polarization. In search of the SLM phase mask that maximizes the overlap integral (OI) between the produced field and the targeted OAM mode, this work aims at achieving the largest possible OI between $E_{f}$ and $U_{f}$. To this end, $U_{f}$ is inverse-propagated through the lens and, ignoring the quarter-wave plate and the aperture, to the SLM plane. The phase of the obtained field is converted into the SLM phase mask, hence used to modulate $E_{s}$. The resulting field is propagated forward to the fiber end, again ignoring the quarter-wave plate and the aperture, yielding $E_{f}$. As proposed in Ref. 6 , the phase mask and the corresponding $E_{f}$ are iteratively calculated for different values of $d_{\ell}$ and $w_{s}$, until the largest coupling between $E_{f}$ and $U_{f}$ is achieved.

This preliminary optimization is referred to as lossless because all the light at the SLM plane is assumed to reach the lens and the fiber end, without being cropped by the boundaries of the calculation domain or by the aperture.

\subsection{Phase Mask Discretization}

The phase mask calculated for the optimal $d_{\ell}$ and $w_{s}$ corresponds to a phase distribution of the form $(\nu \phi+\psi(r))$. $^{*}$ The radial coordinate is discretized into $(N+1)$ values equally spaced by a distance $\Delta_{r}$, obtaining $r_{n}=n \Delta_{r}, n=$

\footnotetext{
${ }^{*}$ To be rigorous, being the phase mask a matrix of pixels, it should not be described by a continuous function. However, this discretization is assumed to be implicit and beside the point of the present discussion.
} 
$0,1, \ldots N$, where $r_{N}$ is the radius of the largest circle inscribable in the SLM screen. The radial function is sampled at $r_{n}, n \in[1, N]$, obtaining $\psi_{n}=\psi\left(r_{n}\right)$. A piecewise constant interpolation is adopted to yield $\psi(r)=\psi_{n}, \forall r \in\left(r_{n-1}, r_{n}\right]$. In other words, the phase mask is discretized into $N$ rings with inner radius $r_{n-1}$ and outer radius $r_{n}, n=1,2, \ldots N$. The number of rings is chosen such that the OI after discretization is reduced by less than $1.5 \%$, hence ensuring that discretization process negligibly affects the resolution of the phase mask. Besides the upper bound imposed by the finite resolution of the SLM, $N$ must be small enough to guarantee that a small rotation of a at least one ring produces a perceptible change in the OI, hence not ascribable to numerical noise.

\subsection{Ring Number Reduction}

As an optional step to further reduce the number of unknowns, the OI resulting from a rotation by $\pi /(4|\nu|)$ rad of one ring at a time is calculated. The result is analyzed to identify whether there exists $\tilde{N}$ such that the absolute value of the relative change in the OI is less than $10^{-6}, \forall n \in[\tilde{N}, N]$. Since the SLM is illuminated by a beam whose power is mainly confined around the screen center, it is clear that the field modulated by the rings with index larger than or equal to $\tilde{N}$ is too weak to visibly affect $E_{f}$ when only one ring is rotated. Nevertheless, the combined contribution of all rings from the $\tilde{N}$ th to the $N$ th is relevant, suggesting that those rings can be considered as a whole. To this intent, $\psi_{n}$ is set equal to $\psi_{\tilde{N}}$ for all $n \in[\tilde{N}, N]$, hence effectively reducing the number of rings from $N$ to $\tilde{N}$.

\subsection{Optimization Algorithm - Lossy Optimization}

The circular aperture between the SLM and the lens is now considered. This allows the optimization algorithm described in this section to scatter outside the aperture opening, within the domain of calculation, and without producing aliasing, part of the light field that gives a negative contribution to the OI. As a result, the proposed optimization algorithm is able to generate a mask that produces an OI exceeding that calculated in Sec. 2.2, despite the loss of resolution due to the mask discretization.

Let $\boldsymbol{\psi}=\left(\psi_{1}, \psi_{2}, \ldots, \psi_{N}\right)$ and $T(\boldsymbol{\psi})$ be the OI between $E_{f}$ and $U_{f}$, given by

$$
T(\boldsymbol{\psi})=\frac{\left|\int_{A} E_{f} U_{f}^{*} \mathrm{~d} A\right|}{\left(\int_{A}\left|E_{f}\right|^{2} \mathrm{~d} A \int_{A}\left|U_{f}\right|^{2} \mathrm{~d} A\right)^{\frac{1}{2}}},
$$

where "*" is the complex conjugate operator and $A$ is the domain at the fiber end plane. The dependence of $T$ on $\boldsymbol{\psi}$ is due to the fact that, at this stage, $E_{f}$ depends only on the choice of the discretized phase mask, fully defined by the vector $\psi$. As in the gradient ascent algorithm, the proposed optimization searches for the optimal point by following the discrete gradient of the objective function. To this end, the quantity $\Gamma_{n}^{ \pm}$is defined as follows:

$$
\Gamma_{n}^{ \pm}=\frac{T\left(\psi_{1}, \ldots, \psi_{n} \pm \Delta_{\psi}, \ldots, \psi_{N}\right)-T\left(\psi_{1}, \ldots, \psi_{n}, \ldots, \psi_{N}\right)}{\Delta_{\psi}},
$$

where $\Delta_{\psi}=\pi / 4 \mathrm{rad}$. The difference quotient $\Gamma_{n}$ is

$$
\Gamma_{n}= \begin{cases}\Gamma_{n}^{+}, & \text {if } \Gamma_{n}^{+}>0 \text { and } \Gamma_{n}^{+} \geq \Gamma_{n}^{-} \\ -\Gamma_{n}^{-}, & \text {if } \Gamma_{n}^{-}>0 \text { and } \Gamma_{n}^{-}>\Gamma_{n}^{+} \\ 0, & \text { otherwise }\end{cases}
$$

The discrete gradient of the OI is defined as the vector

$$
\boldsymbol{\Gamma}=\left(\Gamma_{1}, \Gamma_{2}, \ldots, \Gamma_{N}\right) .
$$

$\boldsymbol{\Gamma}$ represents the direction toward which $\psi$ must move in order to achieve an increased OI. In fact, according to (3) and (4), if a clockwise rotation of the $n$th ring by $\Delta_{\psi} /|\nu|$ gives a positive contribution to the OI, then $\Gamma_{n}=\Gamma_{n}^{+}$, unless a larger positive contribution is obtained by rotating the same ring in the opposite direction, 
in which case $\Gamma_{n}=-\Gamma_{n}^{-}$. If both rotations negatively affect the OI, then $\Gamma_{n}$ is set equal to $0 .^{\dagger}$ Due to the ring reduction previously addressed, for all $n \in[\tilde{N}, N], \Gamma_{n}=\Gamma_{\tilde{N}}$, entailing that all the rings with index equal to or larger than $\tilde{N}$ rotate synchronously by the same angle, hence behaving as a single ring.

In order to optimize $T\left(\boldsymbol{\psi}^{(k)}\right), \boldsymbol{\Gamma}^{(k)}$ is calculated at each iteration and $\boldsymbol{\psi}^{(k)}$ is updated according to the formula

$$
\boldsymbol{\psi}^{(k)}=\boldsymbol{\psi}^{(k-1)}+\alpha \boldsymbol{\Gamma}^{(k)},
$$

where $\alpha>0$ is the learning rate and $k=1,2, \ldots$ is the iteration number. A superscript in parenthesis is added to indicate at which step the corresponding parameter is calculated. $\boldsymbol{\psi}^{(0)}$ is the initial value of $\boldsymbol{\psi}$ obtained after the discretization.

In this context, a phase variation slightly larger than $\pi$ is considered as a change toward $-\pi$, hence in the direction opposite to that indicated by $\Gamma_{n}^{(k)}$. Therefore, the learning rate is chosen such that $\left|\psi_{n}^{(k)}-\psi_{n}^{(k-1)}\right|<$ $\pi, \forall n \in[1, \tilde{N}]$. For this reason, at each iteration, $\alpha$ is initially set equal to $\min _{n \in[1, \tilde{N}]}\left(\Delta_{\psi} /\left|\Gamma_{n}^{(k)}\right|\right)$, hence ensuring that, at each step, every ring is subject to a phase shift within the interval $\left(-\Delta_{\psi}, \Delta_{\psi}\right)$. If $T\left(\boldsymbol{\psi}^{(k)}\right)<T\left(\boldsymbol{\psi}^{(k-1)}\right)$ the learning rate is gradually reduced until the inequality sign is reversed. The algorithm converges if $\alpha$ becomes so small that $\left\|\boldsymbol{\psi}^{(k)}-\boldsymbol{\psi}^{(k-1)}\right\|<0.01 / \tilde{N}$.

The proposed algorithm is able to achieve high purity OAM modes starting from a wide range of arbitrarily chosen initial conditions. Therefore, using the lossless optimization discussed in Sec. 2.2 to calculate the initial parameters and phase mask is optional. However, adopting the calculated $d_{\ell}$ and $w_{s}$ allows to minimize the losses due to the introduced spatial filtering. In addition, the number of iterations required to let the lossy optimization converge can be reduced by using the calculated phase mask as a starting point. Therefore, the lossless optimization remains a valid guideline to design the setup and it is used in this work to calculate the initial conditions of the system before running the lossy-optimization algorithm.

\section{SIMULATION RESULTS}

The proposed optimization algorithm has been tested for different modes and fiber parameters. Here, as an example, a step-index fiber with a core radius of $6 \mu \mathrm{m}$, core refractive index of 1.45 , cladding refractive index of 1.40 , and the $\mathrm{EH}_{2,2}$ mode $(\nu=3)$ are considered. The wavelength in use is $1550 \mathrm{~nm}$. The SLM screen size is $16 \mathrm{~mm} \times 16 \mathrm{~mm}$ with resolution $1024 \times 1024$ pixels. The lens has a focal length of $6 \mathrm{~mm}$. The calculated optimal distance between the SLM and the lens $\left(d_{\ell}\right)$ is $1.659 \mathrm{~m}$ and the optimal beam radius $\left(w_{s}\right)$ is $3.109 \mathrm{~mm}$. The obtained OI is 0.923 . The calculated phase mask and the real parts of $E_{f}$ and $U_{f}$ are displayed in Fig. 2.

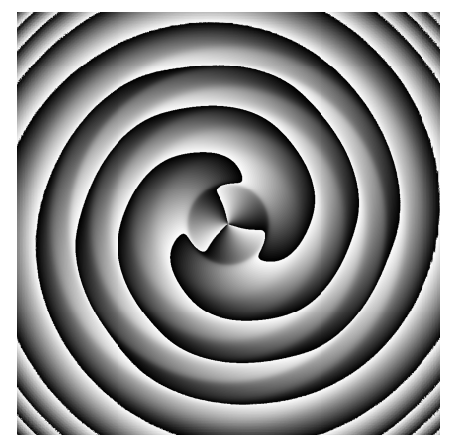

(a)

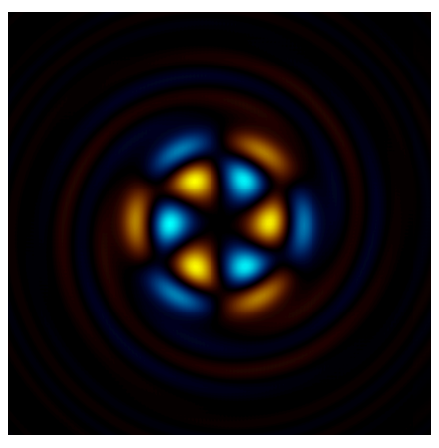

(b)

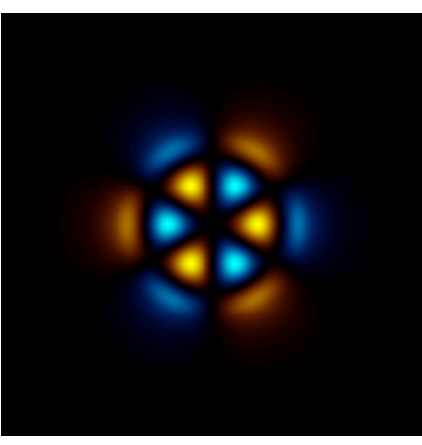

(c)

Figure 2: Ideal conditions for simulations $\left(\mathrm{EH}_{2,2}\right.$ mode). (a) Calculated phase mask at optimal distance, (b) resulting real part of the electric field at the fiber end $\left(E_{f}\right)$, and (c) real part of one polarization component of the electric field of the target mode $\left(U_{f}\right)$.

To emulate a realistic scenario, the starting point is the suboptimal condition achieved by using the calculated phase mask as an initial guess and introducing two errors in the initial parameters $d_{\ell}$ and $w_{s}$. Denoted with a

\footnotetext{
${ }^{\dagger}$ Notice that a ring rotation through $\Delta_{\psi} /|\nu|$ corresponds to a phase change equal to $\Delta_{\psi}$.
} 
bar to avoid confusion, the new values for those parameters are $\bar{d}_{\ell}=0.800 \mathrm{~m}$ and $\bar{w}_{s}=2.200 \mathrm{~mm}$, respectively. As a result, the OI becomes 0.706 . The chosen number of rings $(N)$ is 65 . The calculated $\tilde{N}$ is 48 , obtained by individually rotating each ring through $\pi / 12 \mathrm{rad}$.

\subsection{Simulation 1: Large Aperture Opening}

As following the procedure discussed in Sec. 2.5, a circular aperture is now introduced. For simplicity, the aperture is positioned at the lens plane, hence emulating the clear aperture of the lens itself. The radius of the opening is $5.000 \mathrm{~mm}$. In this example, the presence of the aperture does not affect the OI because its opening is large enough not to noticeably affect the field at the lens plane.

The optimized OI is around 0.937 , achieved after 12 iterations. The optimized phase mask and the real part of $E_{f}$ before and after optimization are shown in Fig. 3. Notice the presence of large discontinuities in the final phase mask (Fig. 3a) which confirms the lossy behavior of the method. The power loss is $1.0 \mathrm{~dB}$, calculated at

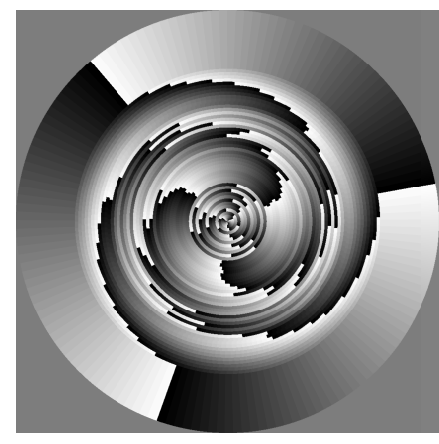

(a)

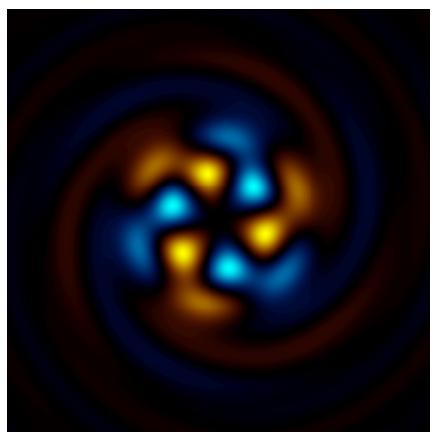

(b)

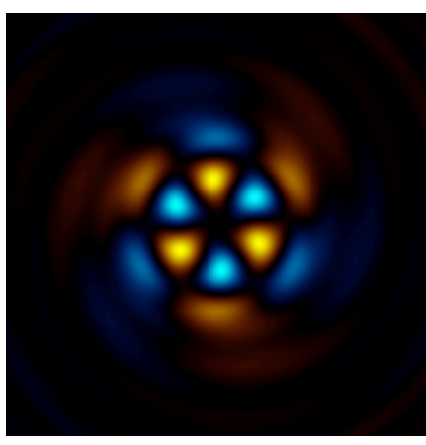

(c)

Figure 3: Results in simulation 1. (a) Optimized phase mask. Real part of the electric field at the fiber end (b) before and (c) after optimization.

the lens plane as the ratio in $\mathrm{dB}$ of the total power reaching the lens to the power in the aperture opening.

\subsection{Simulation 2: Small Aperture Opening}

To show how the the aperture affects the results, a second simulation is performed. The opening radius is now reduced to $2.500 \mathrm{~mm}$. In this case, the initial OI is 0.697 and, after 9 iterations, the optimized OI is 0.972 . The final phase mask and the real part of $E_{f}$ before and after optimization are shown in Fig. 4. Although a

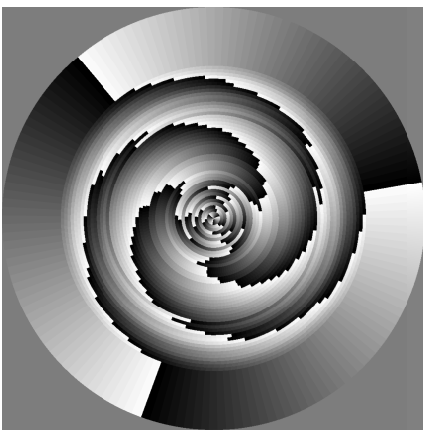

(a)

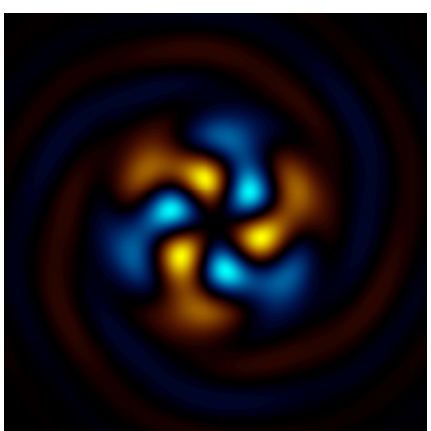

(b)

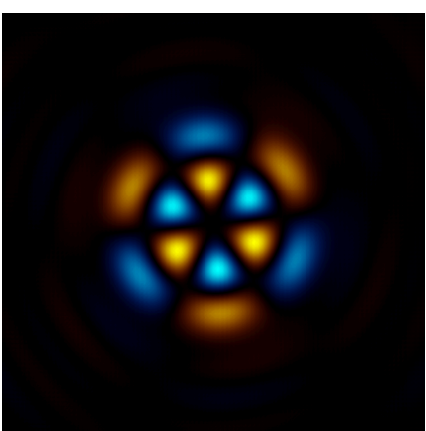

(c)

Figure 4: Results in simulation 2. (a) Optimized phase mask. Real part of the electric field at the fiber end (b) before and (c) after optimization.

clear improvement in the OI is achieved, in this case, the power loss is $2.97 \mathrm{~dB}$, hence much larger than the loss recorded in the first simulation. 


\section{EXPERIMENTAL RESULTS}

Measuring the complex field $E_{f}$ produced experimentally to calculate $T(\psi)$ is a cumbersome task. However, any available technique to evaluate the purity of the excited mode can be equivalently used in place of $T(\boldsymbol{\psi})$ to feed back the proposed optimization algorithm. For example, a time-domain-,${ }^{8}$ imaging-,${ }^{9,10}$ or demultiplexing-based $^{2}$ technique can be adopted. In this experiment, as a test, the interference pattern produced at the lens plane by a reference Gaussian beam and the modulated field is generated. Its comparison with the desired pattern is calculated in the form of OI, which is the objective function of the algorithm.

In general, an interference pattern does not carry enough information about the fields generating it. Hence, it is not possible to retrieve one field by knowing the other. However, in this particular case, extra information is given by the angular phase distribution of the modulated field, which creates an interference pattern that sinusoidally oscillates along the azimuthal coordinate with angular frequency $|\nu|$. Consequently, it is unambiguously possible to imitate the target field at the lens plane by closely reproducing the pattern it generates when interfering with a Gaussian beam. Nevertheless, this technique does not lead to optimal mode purity in the fiber. Its use in this work aims at simplifying the setup and at enabling an easy experimental test.

The wavelength in use is $635 \mathrm{~nm}$. Based on that, the calculation of the optimal setup parameters is performed by simulating a step-index fiber with a core radius of $3 \mu \mathrm{m}$, core refractive index equal to 1.45, and cladding refractive index equal to 1.40. The target mode is again the $\mathrm{CP} \mathrm{EH}_{2,2}$ mode, from which $U_{f}$ is extracted. The simulated lens has a focal length of $4 \mathrm{~mm}$. Under these assumptions, the calculated optimal $d_{\ell}$ and $w_{s}$ are $1.317 \mathrm{~m}$ and $1.792 \mathrm{~mm}$, respectively.

The target interference pattern is calculated as follows. A reference beam, denoted with $E_{\text {ref }}$, is generated by propagating $E_{s}$ (unmodulated) from the SLM plane to the lens plane. $U_{f}$ is inverse-propagated from the fiber end plane to the lens plane and multiplied by the conjugate lens phase, yielding the field named $U_{\ell}$. The interference pattern denoted with $I_{U}$ is defined as follows:

$$
I_{U}=\left|E_{\mathrm{ref}} \exp (i \theta)+U_{\ell}\right|^{3},
$$

where $\theta$ determines the orientation of the interference pattern and is conveniently chosen before starting the optimization process by comparison with the pattern experimentally obtained. Note that $I_{U}$ is proportional to the field intensity raised to the power of $3 / 2$. The use of the third power in (7) in place of the second power aims at obtaining a higher contrast in the pattern, leading to a more efficient optimization, as empirically observed.

The setup for this experiment is depicted in Fig. 5. The laser beam (dotted line) has a wavelength of $635 \mathrm{~nm}$

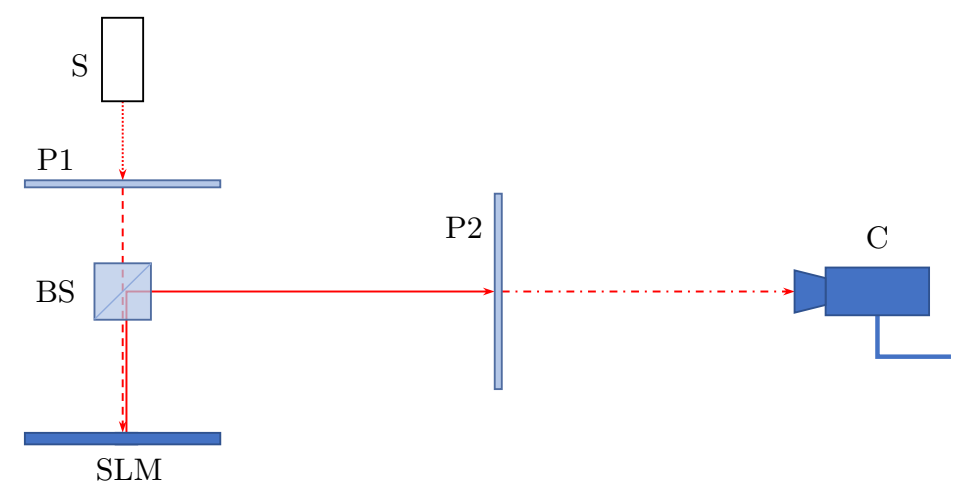

Figure 5: Algorithm test setup. The laser source is indicated by "S." "P1" and "P2" are polarizers, both oriented at $45^{\circ}$ with respect to the direction of the SLM liquid crystal molecules. The beamsplitter is labelled "BS" and the camera with "C." The arrows show the propagation direction of the light beam.

and a $1 / e^{2}$ radius of $\sim 2.3 \mathrm{~mm}$. Since the laser has an unknown initial polarization distribution, a polarizer is used to obtain a beam (dashed line) polarized at $45^{\circ}$ with respect to the direction of the SLM liquid crystal molecules, used as a reference axis. The SLM (Santec SLM-200, $1920 \times 1200$ pixels, $8 \mu \mathrm{m}$ pixel pitch) is illuminated by 
the resulting beam, whose component reflected by the 50/50 beamsplitter (BS) is neglected. In fact, in this context, the BS is used only to illuminate the SLM at a normal angle. The beam reflected by the SLM (solid line) is the superposition of a modulated component polarized at $0^{\circ}$ and an unmodulated component at $90^{\circ}$. A second polarizer oriented at $45^{\circ}$ is placed between the BS and the camera. This polarizer projects both beam components onto its axis, allowing the interference to take place. The resulting beam (dash-dotted line) impinges on the CCD camera (Point Grey, $1920 \times 1440$ pixels, $4.54 \mu \mathrm{m}$ pixel size), hence capturing the sought interference pattern. The camera is located at an optical distance $\sim 1.5 \mathrm{~m}$ away from the SLM, which is where the lens in the mode-excitation setup (Fig. 1) is virtually placed. In order to match the third power in the definition of $I_{U}$, the intensity distribution captured by the camera is raised to the power of $3 / 2$. The result is denoted by $I_{E}$.

The OI between $I_{E}$ and $I_{U}$, derived from (2), is defined as follows:

$$
T_{I}(\psi)=\frac{\int_{S} I_{E} I_{U} \mathrm{~d} S}{\left(\int_{S} I_{E}^{2} \mathrm{~d} S \int_{S} I_{U}^{2} \mathrm{~d} S\right)^{\frac{1}{2}}},
$$

where $S$ is the domain at the lens plane, defined as a circle whose radius is $2.250 \mathrm{~mm}$, hence emulating the spatial filtering due to an aperture with the same radius. $T_{I}$ is minimized using the algorithm described in Sec. 2.5, substituting $T$ for $T_{I}$ in (3). The values of $N$ and $\tilde{N}$ are both equal to 75 . This is due to the fact that the beam decays too slowly over the SLM screen. As a result, the rotation of none of the outer rings gives a negligible contribution to the OI. This also means that the field strength at the edges of SLM screen is strong enough to break the circular symmetry of the system. Despite that, the optimization successfully converges to a high value of $T_{I}$, equal to 0.942 , achieved after 5 iterations, hence demonstrating the robustness of the proposed algorithm.

The initial $I_{E}$ and its evolution throughout the simulation are displayed in Fig. 6, along with the target interference pattern $I_{U}$.

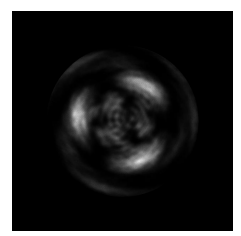

(a)

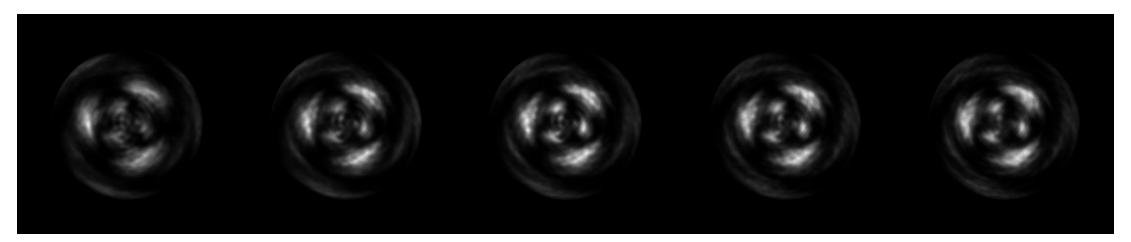

(b)

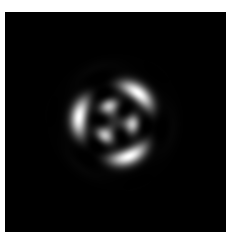

(c)

Figure 6: Experimental result. (a) Initial interference pattern $\left(T_{I}=0.769\right)$. (b) Interference pattern obtained at the end of each iteration, in chronological order from left to right. Respectively, the corresponding values of $T_{I}$ are $0.895,0.926,0.938,0.941,0.942$. (c) Target interference pattern.

\section{CONCLUSION}

In this work, a method to excite high-purity orbital angular momentum fiber modes has been developed. The proposed mode-excitation setup consists of one phase-only spatial light modulator (SLM), a circular aperture, and a lens. The problem of iteratively calculating the SLM phase mask that maximizes the purity of the target mode has been addressed. The proposed approach is based on a previous work, wherein the SLM phase mask is considered as a collection of $N$ contiguous annular regions, each of which is defined by a real number $\psi_{n}, n=1,2, \ldots, N$, representing the angular position of the $n$th ring. The proposed algorithm finds the values of $\psi_{n}$ that maximize the mode purity. The optimization process relies on the high diffraction that the algorithm spontaneously generates to filter out of the aperture opening portions of light which reduce the mode purity. Simulations and experimental results have shown the effectiveness and the robustness of the proposed algorithm.

\section{ACKNOWLEDGMENTS}

The Authors would like to thank Santec for the technical support.

This project has received funding from the European Union's Horizon 2020 research and innovation programme under the Marie Sklodowska-Curie grant agreement No. 642355. 


\section{REFERENCES}

[1] Ramachandran, S. and Kristensen, P., "Optical vortices in fiber," Nanophotonics 2(5-6), 455-474 (2013).

[2] Ingerslev, K., Gregg, P., Galili, M., Ros, F. D., Hu, H., Bao, F., Castaneda, M. A. U., Kristensen, P., Rubano, A., Marrucci, L., Ramachandran, S., Rottwitt, K., Morioka, T., and Oxenlowe, L. K., "12 mode, MIMO-free OAM transmission," in [Optical Fiber Communication Conference], Optical Fiber Communication Conference, M2D.1, Optical Society of America (2017).

[3] Nejad, R. M., Allahverdyan, K., Vaity, P., Amiralizadeh, S., Brunet, C., Messaddeq, Y., LaRochelle, S., and Rusch, L. A., "Mode division multiplexing using orbital angular momentum modes over 1.4-km ring core fiber," Journal of Lightwave Technology 34(18), 4252-4258 (2016).

[4] Bozinovic, N., Yue, Y., Ren, Y., Tur, M., Kristensen, P., Huang, H., Willner, A. E., and Ramachandran, S., "Terabit-scale orbital angular momentum mode division multiplexing in fibers," Science 340(6140), 1545-1548 (2013).

[5] Astorino, A., Glückstad, J., and Rottwitt, K., "Towards the use of machine learning in setups for oam mode excitation in optical fibers," in [Frontiers in Optics], JTu2A-6, Optical Society of America (2018).

[6] Astorino, A., Glückstad, J., and Rottwitt, K., "Fiber mode excitation using phase-only spatial light modulation: Guideline on free-space path design and lossless optimization," AIP Advances 8(9), 095111 (2018).

[7] Astorino, A., Lægsgaard, J., and Rottwitt, K., "The bowtie effect in cylindrical waveguides," Journal of Lightwave Technology 36(16), 3309-3317 (2018).

[8] Nakazawa, M., Yoshida, M., and Hirooka, T., "Nondestructive measurement of mode couplings along a multi-core fiber using a synchronous multi-channel OTDR," Optics Express 20(11), 12530-12540 (2012).

[9] Nicholson, J. W., Yablon, A. D., Ramachandran, S., and Ghalmi, S., "Spatially and spectrally resolved imaging of modal content in large-mode-area fibers," Optics Express 16(10), 7233-7243 (2008).

[10] Schimpf, D. N., Barankov, R. A., and Ramachandran, S., "Cross-correlated (C-2) imaging of fiber and waveguide modes," Optics Express 19(14), 13008-13019 (2011). 